\title{
Discrete Weighted Pseudo Asymptotic Periodicity of Second Order Difference Equations
}

\author{
Zhinan Xia \\ Department of Applied Mathematics, Zhejiang University of Technology, Hangzhou, Zhejiang 310023, China \\ Correspondence should be addressed to Zhinan Xia; xiazn299@zjut.edu.cn
}

Received 17 March 2014; Accepted 21 July 2014; Published 10 August 2014

Academic Editor: Zhan Zhou

Copyright (C) 2014 Zhinan Xia. This is an open access article distributed under the Creative Commons Attribution License, which permits unrestricted use, distribution, and reproduction in any medium, provided the original work is properly cited.

\begin{abstract}
We define the concept of discrete weighted pseudo- $\mathcal{\delta}$-asymptotically periodic function and prove some basic results including composition theorem. We investigate the existence, and uniqueness of discrete weighted pseudo- $\mathcal{\delta}$-asymptotically periodic solution to nonautonomous semilinear difference equations. Furthermore, an application to scalar second order difference equations is given. The working tools are based on the exponential dichotomy theory and fixed point theorem.
\end{abstract}

\section{Introduction}

The concept of $\mathcal{S}$-asymptotic periodicity $[1,2]$ introduced by Henríquez et al. is natural generalization of asymptotic periodicity [3]. Since then, many contributions on the existence of $\delta$-asymptotically periodic solutions for differential equations have been made; one can see [4-10] for more details. Further Pierri and Rolnik [11] introduced the notion of pseudo- $\delta$-asymptotically periodic function, explored its properties, and investigated pseudo- $\mathcal{S}$-asymptotic periodicity of neutral differential equations with finite delay in Banach space. With the help of weighted function, weighted pseudo$\mathcal{S}$-asymptotically periodic function is introduced and the applications to fractional integrodifferential equations are investigated in [12].

All the above-mentioned concepts are introduced in the continuous case, but it is rarely for the discrete type. For (discrete) $\delta$-asymptotic periodicity, the subject has been studied in the recent paper [13], where the authors discussed the existence of (discrete) $\mathcal{S}$-asymptotically periodic solutions of semilinear difference equations with infinite delay. Motivated by the above literatures, it is natural to consider the discrete version of weighted pseudo- $\mathcal{S}$-asymptotically periodic function, which we will discuss in the present paper.

The rapid development of the theory of difference equations has been strongly promoted by the large number of applications in physics, engineering, biology, and other subjects. The asymptotic behaviour of solutions of difference equations is at present an active of research. Many researchers have made important contributions to these topics, for example, almost periodicity $[14,15]$, asymptotic almost periodicity $[16,17]$, almost automorphy [18-20], $l^{p}$-boundedness [21], stability [22, 23], and $\delta$-asymptotic periodicity [13]. However, to the best of our knowledge, (discrete) weighted pseudo- $\mathcal{S}$ asymptotic periodicity of difference equations is quite new and an untreated topic. This is one of the key motivations of this study.

The principal aim of this paper is to introduce the concept of discrete weighted pseudo- $\mathcal{S}$-asymptotically periodic function, which is much more general to generalize discrete $\mathcal{S}$-asymptotically periodic function and explore its properties and applications in difference equations. The paper is organized as follows. In Section 2, first, some notations and preliminary results are presented. Next, we propose a new class of functions called discrete weighted pseudo- $\mathcal{\delta}$-asymptotically $\omega$-periodic function, explore its properties, and establish the composition theorem. Section 3 is devoted to the existence and uniqueness of discrete weighted pseudo- $\mathcal{S}$ asymptotically $\omega$-periodic solution of nonautonomous semilinear difference equations. In Section 4, discrete weighted pseudo- $\delta$-asymptotic $\omega$-periodicity of the scalar second order difference equations is investigated. 


\section{Preliminaries and Basic Results}

Let $(X,\|\cdot\|),(Y,\|\cdot\|)$ be two Banach spaces and let $\mathbb{N}$, $\mathbb{Z}, \mathbb{Z}^{+} \mathbb{R}, \mathbb{R}^{+}$, and $\mathbb{C}$ stand for the set of natural numbers, integers, nonnegative integers, real numbers, nonnegative real numbers, and complex numbers, respectively. Let $A$ be bounded linear operator; $\sigma_{p}(A)$ denotes the point spectrum of $A$.

In order to facilitate the discussion below, we further introduce the following notations.

(i) $S^{1}=\{\lambda \in \mathbb{C},|\lambda|=1\}$.

(ii) $l^{\infty}(\mathbb{Z}, X)=\left\{x: \mathbb{Z} \rightarrow X:\|x\|_{d}:=\sup _{n \in \mathbb{Z}}\|x(n)\|<\right.$ $\infty\}$.

(iii) $C_{0}(\mathbb{Z}, X)=\left\{x \in l^{\infty}(\mathbb{Z}, X): \lim _{|n| \rightarrow \infty}\|x(n)\|=0\right\}$.

(iv) $C_{\omega}(\mathbb{Z}, X)=\left\{x \in l^{\infty}(\mathbb{Z}, X): x\right.$ is $\omega$-priodic $\}$, where $\omega \in \mathbb{Z}^{+} \backslash\{0\}$.

(v) $L(X, Y)$ is the Banach space of bounded linear operators from $X$ to $Y$ endowed with the operator topology. In particular, we write $L(X)$ when $X=Y$.

(vi) $\mathcal{U} \mathscr{C}(\mathbb{Z} \times X, X)$ is the set of all functions $f: \mathbb{Z} \times X \rightarrow$ $X$ satisfying that $\forall \varepsilon>0, \exists \delta>0$ such that

$$
\|f(k, x)-f(k, y)\| \leq \varepsilon
$$

for all $k \in \mathbb{Z}$ and $x, y \in X$ with $\|x-y\| \leq \delta$.

First, we recall the so-called Matkowski's fixed point theorem [24] and exponential dichotomy [25] which will be used in the sequel.

Theorem 1 (Matkowski's fixed point theorem [24]). Let $(X, d)$ be a complete metric space and let $\mathscr{F}: X \rightarrow X$ be a map such that

$$
d(\mathscr{F} x, \mathscr{F} y) \leq \Phi(d(x, y)), \quad \forall x, y \in X
$$

where $\Phi:[0, \infty) \rightarrow[0, \infty)$ is a nondecreasing function such that $\lim _{n \rightarrow \infty} \Phi^{n}(t)=0$ for all $t>0$. Then $\mathscr{F}$ has a unique fixed point $z \in X$.

Given a sequence $\{A(n)\}_{n \in \mathbb{Z}} \subset L(X)$ of invertible operators, define

$$
\mathscr{A}(m, n)= \begin{cases}A(m-1) \cdots A(n), & \text { if } m>n, \\ \mathrm{Id}, & \text { if } m=n, \\ A^{-1}(m) \cdots A^{-1}(n-1), & \text { if } m<n,\end{cases}
$$

where Id is the identity operator in $X$.

For the first order difference equation

$$
x(n+1)=A(n) x(n), \quad n \in \mathbb{Z} .
$$

Definition 2 (see [25]). Equation (4) is said to have an exponential dichotomy if there exist projections $P(n) \in L(X)$ for all $n \in \mathbb{Z}$ and positive constants $\eta, \nu, \alpha, \beta$ such that

(i) $P(m) \mathscr{A}(m, n)=\mathscr{A}(m, n) P(n), m, n \in \mathbb{N}$,

(ii) $\|\mathscr{A}(m, n) P(n)\| \leq \eta e^{-\alpha(m-n)}, m \geq n$, (iii) $\|\mathscr{A}(m, n) Q(n)\| \leq v e^{-\beta(n-m)}, n \geq m$,

where $Q(n)=\mathrm{Id}-P(n)$ is the complementary projection of $P(n)$.

Next, we propose a new class of functions called discrete weighted pseudo- $\mathcal{S}$-asymptotically $\omega$-periodic function and explore its properties including composition result.

Let $U$ denote the collection of functions (weights) $\rho$ : $\mathbb{Z} \rightarrow(0,+\infty)$. For $\rho \in U$ and $n \in \mathbb{Z}^{+}$, set

$$
\mu(n, \rho):=\sum_{k=-n}^{n} \rho(k) .
$$

Denote

$$
\begin{gathered}
U_{\infty}:=\left\{\rho \in U: \lim _{n \rightarrow \infty} \mu(n, \rho)=\infty\right\}, \\
U_{B}:=\left\{\rho \in U_{\infty}: 0<\inf _{k \in \mathbb{Z}} \rho(k) \leq \sup _{k \in \mathbb{Z}} \rho(k)<\infty\right\} .
\end{gathered}
$$

Definition 3. Let $\rho_{1}, \rho_{2} \in U_{\infty}$. $\rho_{1}$ is said to be equivalent to $\rho_{2}$ (i.e., $\left.\rho_{1} \sim \rho_{2}\right)$ if $\left(\rho_{1} / \rho_{2}\right) \in U_{B}$.

It is trivial to show that " $\sim$ " is a binary equivalence relation on $U_{\infty}$. The equivalence class of a given weight $\rho \in U_{\infty}$ is denoted by $\operatorname{cl}(\rho)=\left\{\varrho \in U_{\infty}: \rho \sim \varrho\right\}$. It is clear that $U_{\infty}=$ $\bigcup_{\rho \in U_{\infty}} \operatorname{cl}(\rho)$.

Let $\rho \in U_{\infty}, m \in \mathbb{Z}$; define $\rho_{m}$ by $\rho_{m}(n)=\rho(n+m)$ for $n \in \mathbb{Z}$ and

$$
U_{T}=\left\{\rho \in U_{\infty}: \rho \sim \rho_{m} \text { for each } m \in \mathbb{Z}\right\} .
$$

Definition 4. A function $f \in l^{\infty}(\mathbb{Z}, X)$ is called discrete asymptotically $\omega$-periodic if there exist $g \in C_{\omega}(\mathbb{Z}, X), \varphi \in$ $C_{0}(\mathbb{Z}, X)$ such that $f=g+\varphi$. The collection of those functions is denoted by $A P_{\omega}(\mathbb{Z}, X)$.

Definition 5. A function $f \in l^{\infty}(\mathbb{Z}, X)$ is called discrete $\mathcal{S}$ asymptotically $\omega$-periodic if there exists $\omega \in \mathbb{Z}^{+} \backslash\{0\}$ such that $\lim _{n \rightarrow \infty}(f(n+\omega)-f(n))=0$. The collection of those functions is denoted by $\mathcal{S} A P_{\omega}(\mathbb{Z}, X)$.

Definition 6. A function $f \in l^{\infty}(\mathbb{Z}, X)$ is called discrete pseudo- $\mathcal{S}$-asymptotically $\omega$-periodic if there exists $\omega \in \mathbb{Z}^{+} \mid$ $\{0\}$ such that

$$
\lim _{n \rightarrow \infty} \frac{1}{2 n} \sum_{k=-n}^{n}\|f(k+\omega)-f(k)\|=0 .
$$

Denote by $P \mathcal{S} A P_{\omega}(\mathbb{Z}, X)$ the set of such functions.

Definition 7. Let $\rho \in U_{\infty}$. A function $f \in l^{\infty}(\mathbb{Z}, X)$ is called discrete weighted pseudo- $\mathcal{S}$-asymptotically $\omega$-periodic if there exists $\omega \in \mathbb{Z}^{+} \backslash\{0\}$ such that

$$
\lim _{n \rightarrow \infty} \frac{1}{\mu(n, \rho)} \sum_{k=-n}^{n} \rho(k)\|f(k+\omega)-f(k)\|=0 .
$$

Denote by $W P \mathcal{S} A P_{\omega}(\mathbb{Z}, X, \rho)$ the set of such functions. 
Next, we will show some properties of $W P \mathcal{S} A P_{\omega}(\mathbb{Z}, X, \rho)$ including composition theorem.

Lemma 8. Let $\rho \in U_{\infty}$; then the following properties hold:

(i) $f \pm g \in W P S A P_{\omega}(\mathbb{Z}, X, \rho)$ if $f, g \in W P S A P_{\omega}(\mathbb{Z}$, $X, \rho)$;

(ii) $\lambda f \in W P S A P_{\omega}(\mathbb{Z}, X, \rho)$ if $\lambda \in \mathbb{Z}, f \in W P S A P_{\omega}(\mathbb{Z}$, $X, \rho)$;

(iii) $A P_{\omega}(\mathbb{Z}, X) \subset \mathcal{S A P} P_{\omega}(\mathbb{Z}, X) \subset P \mathcal{S} A P_{\omega}(\mathbb{Z}, X) \subset$ $W P S A P_{\omega}(\mathbb{Z}, X, \rho)$;

(iv) $W P S A P_{\omega}(\mathbb{Z}, X, \rho)$ is a Banach space when endowed with the sup norm

$$
\|f\|_{d}:=\sup _{n \in \mathbb{Z}}\|f(n)\| .
$$

Proof. The proof is straightforward, so the details are omitted here.

Lemma 9. Assume that $\rho_{1}, \rho_{2} \in U_{\infty}$. If $\rho_{1} \sim \rho_{2}$, then

(i) $W P S A P_{\omega}\left(\mathbb{Z}, X, \rho_{1}\right)=W P S A P_{\omega}\left(\mathbb{Z}, X, \rho_{2}\right)$;

(ii) $W P S A P_{\omega}\left(\mathbb{Z}, X, \rho_{1}+\rho_{2}\right)=W P \mathcal{S A P} P_{\omega}\left(\mathbb{Z}, X, \rho_{1}\right)=$ $W P S A P_{\omega}\left(\mathbb{Z}, X, \rho_{2}\right)$;

(iii) $W P S A P_{\omega}\left(\mathbb{Z}, X, \rho_{1} / \rho_{2}\right)=W P S A P_{\omega}(\mathbb{Z}, X, c l(1))=$ $P \operatorname{SAP}_{\omega}(\mathbb{Z}, X)$

(iv) if $\rho \in U_{B}$, then $W P \mathcal{S} A P_{\omega}(\mathbb{Z}, X, \rho)=P \mathcal{S} A P_{\omega}(\mathbb{Z}, X)$.

Proof. (i) Since $\rho_{1} \sim \rho_{2}$, there exist $K_{1}>0$ and $K_{2}>0$ such that $K_{1} \rho_{2} \leq \rho_{1} \leq K_{2} \rho_{2}$; then

$$
K_{1} \mu\left(n, \rho_{2}\right) \leq \mu\left(n, \rho_{1}\right) \leq K_{2} \mu\left(n, \rho_{2}\right) .
$$

Let $f \in W P \mathcal{S A P}\left(\mathbb{Z}, X, \rho_{2}\right)$; then

$$
\begin{aligned}
& \frac{1}{\mu\left(n, \rho_{1}\right)} \sum_{k=-n}^{n} \rho_{1}(k)\|f(k+\omega)-f(k)\| \\
& =\frac{1}{\mu\left(n, \rho_{1}\right)} \sum_{k=-n}^{n} \frac{\rho_{1}(k)}{\rho_{2}(k)} \rho_{2}(k)\|f(k+\omega)-f(k)\| \\
& \leq \frac{K_{2}}{\mu\left(n, \rho_{1}\right)} \sum_{k=-n}^{n} \rho_{2}(k)\|f(k+\omega)-f(k)\| \\
& \leq \frac{K_{2}}{K_{1} \mu\left(n, \rho_{2}\right)} \sum_{k=-n}^{n} \rho_{2}(k)\|f(k+\omega)-f(k)\| .
\end{aligned}
$$

The fact that $f \in W P \mathcal{S} A P_{\omega}\left(\mathbb{Z}, X, \rho_{2}\right)$ implies that

$$
\lim _{n \rightarrow \infty} \frac{1}{\mu\left(n, \rho_{2}\right)} \sum_{k=-n}^{n} \rho_{2}(k)\|f(k+\omega)-f(k)\|=0 ;
$$

then

$$
\lim _{n \rightarrow \infty} \frac{1}{\mu\left(n, \rho_{1}\right)} \sum_{k=-n}^{n} \rho_{1}(k)\|f(k+\omega)-f(k)\|=0 ;
$$

that is, $f \in W P S A P_{\omega}\left(\mathbb{Z}, X, \rho_{1}\right)$; hence

$$
W P S A P_{\omega}\left(\mathbb{Z}, X, \rho_{2}\right) \subset W P S A P_{\omega}\left(\mathbb{Z}, X, \rho_{1}\right) .
$$

Proceeding in a similar manner, we have $\operatorname{WPS}_{\mathcal{S}} A P_{\omega}(\mathbb{Z}, X$, $\left.\rho_{1}\right) \subset W P \mathcal{S} A P_{\omega}\left(\mathbb{Z}, X, \rho_{2}\right)$. Hence (i) holds.

From the proof of (i), it is not difficult to see that (ii), (iii), and (iv) hold, so the details are omitted here. The proof is completed.

Lemma 10. Let $f \in W P S A P_{\omega}(\mathbb{Z}, X, \rho), \rho \in U_{T}$; then $f(\cdot+$ $m) \in W P S A P_{\omega}(\mathbb{Z}, X, \rho)$ for all $m \in \mathbb{Z}$.

Proof. Without loss of generality, we may assume that $m \in$ $\mathbb{Z}^{+}$,

$$
\begin{aligned}
& \frac{1}{\mu(n, \rho)} \sum_{k=-n}^{n} \rho(k)\|f(k+m+\omega)-f(k+m)\| \\
& =\frac{1}{\mu(n, \rho)} \sum_{k=-n+m}^{n+m} \rho_{-m}(k)\|f(k+\omega)-f(k)\| \\
& \leq \frac{\mu(n+m, \rho)}{\mu(n, \rho)} \frac{1}{\mu(n+m, \rho)} \\
& \quad \times \sum_{k=-n-m}^{n+m} \frac{\rho_{-m}(k)}{\rho(k)} \rho(k)\|f(k+\omega)-f(k)\|,
\end{aligned}
$$

since $\rho \in U_{T}$ implies that there exists $\kappa>0$ such that $\rho_{m}(k) / \rho(k) \leq \kappa, \rho_{-m}(k) / \rho(k) \leq \kappa$. For $n>m$

$$
\begin{aligned}
\mu(n+m, \rho) & =\sum_{k=-n-m}^{n+m} \rho(k)=\sum_{k=-n-m}^{n-m} \rho(k)+\sum_{k=n-m}^{n+m} \rho(k) \\
& \leq \sum_{k=-n-m}^{n-m} \rho(k)+\sum_{k=-n+m}^{n+m} \rho(k) \\
& =\sum_{k=-n}^{n} \rho_{-m}(k)+\sum_{k=-n}^{n} \rho_{m}(k) \leq 2 \kappa \mu(n, \rho) ;
\end{aligned}
$$

then

$$
\begin{aligned}
& \frac{1}{\mu(n, \rho)} \sum_{k=-n}^{n} \rho(k)\|f(k+m+\omega)-f(k+m)\| \\
& \leq \frac{2 \kappa^{2}}{\mu(n+m, \rho)} \sum_{k=-n-m}^{n+m} \rho(k)\|f(k+\omega)-f(k)\| .
\end{aligned}
$$

Note that $f \in W P \mathcal{S} A P_{\omega}(\mathbb{Z}, X, \rho), \rho \in U_{T}$; therefore $f(\cdot+m) \in$ $W P S A P_{\omega}(\mathbb{Z}, X, \rho)$.

We will establish composition theorem for discrete weighted pseudo- $\mathcal{S}$-asymptotically $\omega$-periodic function.

Lemma 11. Let $f \in l^{\infty}(\mathbb{Z}, X)$; then $f \in W P \mathcal{S} A P_{\omega}(\mathbb{Z}, X, \rho)$, $\rho \in U_{\infty}$, if and only if, for any $\varepsilon>0$,

$$
\lim _{n \rightarrow \infty} \frac{1}{\mu(n, \rho)} \sum_{k \in E_{f}(n, \varepsilon)} \rho(k)=0,
$$

where $E_{f}(n, \varepsilon)=\{k \in[-n, n] \cap \mathbb{Z}:\|f(k+\omega)-f(k)\| \geq \varepsilon\}$. 
Proof. Sufficiency: it is clear that $M=\sup _{n \in \mathbb{Z}}\|f(n)\|<\infty$ and $\forall \varepsilon>0$; there exists $N \in \mathbb{N}$ such that

$$
\frac{1}{\mu(n, \rho)} \sum_{k \in E_{f}(n, \varepsilon)} \rho(k)<\frac{\varepsilon}{2 M}, \quad n>N .
$$

Then, for $n>N$,

$$
\begin{aligned}
& \frac{1}{\mu(n, \rho)} \sum_{k=-n}^{n} \rho(k)\|f(k+\omega)-f(k)\| \\
& =\frac{1}{\mu(n, \rho)} \sum_{k \in E_{f}(n, \varepsilon)} \rho(k)\|f(k+\omega)-f(k)\| \\
& \quad+\frac{1}{\mu(n, \rho)} \sum_{k \in([-n, n] \cap \mathbb{Z}) \backslash E_{f}(n, \varepsilon)} \rho(k)\|f(k+\omega)-f(k)\| \\
& \leq 2 M \cdot \frac{1}{\mu(n, \rho)} \sum_{k \in E_{f}(n, \varepsilon)} \rho(k) \\
& \quad+\varepsilon \cdot \frac{1}{\mu(n, \rho)} \sum_{k \in([-n, n] \cap \mathbb{Z}) \backslash E_{f}(n, \varepsilon)} \rho(k) \leq 2 \varepsilon,
\end{aligned}
$$

so

$$
\lim _{n \rightarrow \infty} \frac{1}{\mu(n, \rho)} \sum_{k=-n}^{n} \rho(k)\|f(k+\omega)-f(k)\|=0 .
$$

That is, $f \in W P \mathcal{S} A P_{\omega}(\mathbb{Z}, X, \rho)$.

Necessity: suppose the contrary that there exists $\varepsilon_{0}>0$, such that $(1 / \mu(n, \rho)) \sum_{k \in E_{f}(n, \varepsilon)} \rho(k)$ does not converge to 0 as $n \rightarrow \infty$. That is, there exists $\delta>0$, such that, for each $m \in \mathbb{Z}$,

$$
\frac{1}{\mu\left(n_{m}, \rho\right)} \sum_{k \in E_{f}\left(n_{m}, \varepsilon_{0}\right)} \rho(k) \geq \delta, \quad \text { for some } n_{m}>m \text {. }
$$

Then for $n_{m}>m$

$$
\begin{aligned}
& \frac{1}{\mu\left(n_{m}, \rho\right)} \sum_{k=-n_{m}}^{n_{m}} \rho(k)\|f(k+\omega)-f(k)\| \\
& =\frac{1}{\mu\left(n_{m}, \rho\right)} \sum_{k \in E_{f}\left(n_{m}, \varepsilon_{0}\right)} \rho(k)\|f(k+\omega)-f(k)\| \\
& \quad+\frac{1}{\mu\left(n_{m}, \rho\right)} \sum_{k \in\left(\left[-n_{m}, n_{m}\right] \cap \mathbb{Z}\right) \backslash E_{f}\left(n_{m}, \varepsilon_{0}\right)} \rho(k)\|f(k+\omega)-f(k)\| \\
& \geq \frac{1}{\mu\left(n_{m}, \rho\right)} \sum_{k \in E_{f}\left(n_{m}, \varepsilon_{0}\right)} \rho(k)\|f(k+\omega)-f(k)\| \\
& \geq \frac{\varepsilon_{0}}{\mu\left(n_{m}, \rho\right)} \sum_{k \in E_{f}\left(n_{m}, \varepsilon_{0}\right)} \rho(k) \geq \delta \varepsilon_{0},
\end{aligned}
$$

which contradicts the fact that

$$
\lim _{n \rightarrow \infty} \frac{1}{\mu(n, \rho)} \sum_{k=-n}^{n} \rho(k)\|f(k+\omega)-f(k)\|=0 .
$$

Thus (19) holds.
Theorem 12. Let $\rho \in U_{\infty}$. Assume that $f \in W P \mathcal{S} A P_{\omega}(\mathbb{Z} \times$ $X, X, \rho) \cap \mathscr{U} \mathscr{C}(\mathbb{Z} \times X, X)$; then $\psi(\cdot)=f(\cdot, u(\cdot)) \in$ $W_{P S A P}(\mathbb{Z}, X, \rho)$ if $u \in W P \mathcal{S} A P_{\omega}(\mathbb{Z}, X, \rho)$.

Proof. Since $f \in \mathscr{U} \mathscr{C}(\mathbb{Z} \times X, X)$, for any $\varepsilon>0$, there exists $\delta>0$ such that

$$
\|f(k, u(k+\omega))-f(k, u(k))\| \leq L_{f} \mathcal{E}
$$

for all $k \in \mathbb{Z}$ and $\|u(k+\omega)-u(k)\| \leq \delta$. For the above $\varepsilon>0$, since $f \in W P \mathcal{S} A P_{\omega}(\mathbb{Z} \times X, X, \rho)$, there exists $N \in \mathbb{N}$ such that, for $n>N$,

$$
\frac{1}{\mu(n, \rho)} \sum_{k=-n}^{n} \rho(k)\|f(k+\omega, x)-f(k, x)\| \leq \varepsilon
$$

for each $x \in X$.

Denote

$$
E_{u}(n, \delta)=\{k \in[-n, n] \cap \mathbb{Z}:\|u(k+\omega)-u(k)\| \geq \delta\} ;
$$

then $\lim _{n \rightarrow \infty}(1 / \mu(n, \rho)) \sum_{k \in E_{u}(n, \delta)} \rho(k)=0$ by Lemma 11. So

$$
\begin{aligned}
& \frac{1}{\mu(n, \rho)} \sum_{k=-n}^{n} \rho(k)\|f(k, u(k+\omega))-f(k, u(k))\| \\
& =\frac{1}{\mu(n, \rho)} \sum_{k \in E_{u}(n, \delta)} \rho(k)\|f(k, u(k+\omega))-f(k, u(k))\| \\
& \quad+\frac{1}{\mu(n, \rho)} \\
& \quad \times \sum_{k \in([-n, n] \cap \mathbb{Z}) \backslash E_{u}(n, \delta)} \rho(k)\|f(k, u(k+\omega))-f(k, u(k))\| \\
& \leq 2\|\psi\|_{d} \cdot \frac{1}{\mu(n, \rho)} \sum_{k \in E_{u}(n, \delta)} \rho(k) \\
& \quad+\frac{1}{\mu(n, \rho)} \sum_{k \in([-n, n] \cap \mathbb{Z}) \backslash E_{u}(n, \delta)} \rho(k) L_{f} \mathcal{E} \\
& \leq 2\|\psi\|_{d} \cdot \frac{1}{\mu(n, \rho)} \sum_{k \in E_{u}(n, \delta)} \rho(k)+\frac{1}{\mu(n, \rho)} \sum_{k=-n}^{n} \rho(k) L_{f} \mathcal{E} \\
& \leq 2\|\psi\|_{d} \cdot \frac{1}{\mu(n, \rho)} \sum_{k \in E_{u}(n, \delta)} \rho(k)+L_{f} \mathcal{E},
\end{aligned}
$$

where $\|\psi\|_{d}=\sup _{n \in \mathbb{Z}}\|\psi(n)\|$. 
For $n>N$, one has

$$
\begin{aligned}
& \frac{1}{\mu(n, \rho)} \sum_{k=-n}^{n} \rho(k)\|f(k+\omega, u(k+\omega))-f(k, u(k))\| \\
& \leq \frac{1}{\mu(n, \rho)} \sum_{k=-n}^{n} \rho(k) \| f(k+\omega, u(k+\omega)) \\
& \quad-\frac{1}{\mu(n, \rho)} \sum_{k=-n}^{n} \rho(k)\|f(k, u(k+\omega))-f(k, u(k))\| \\
& \leq \varepsilon+2\|\psi\|_{d} \cdot \frac{1}{\mu(n, \rho)} \sum_{k \in E_{u}(n, \delta)} \rho(k)+L_{f} \mathcal{E} .
\end{aligned}
$$

Due to the arbitrariness of $\varepsilon$, one has

$$
\begin{aligned}
\lim _{n \rightarrow \infty} & \frac{1}{\mu(n, \rho)} \sum_{k=-n}^{n} \rho(k)\|f(k+\omega, u(k+\omega))-f(k, u(k))\| \\
& =0
\end{aligned}
$$

which implies that $\psi(\cdot) \in W P S A P_{\omega}(\mathbb{Z}, X, \rho)$.

Corollary 13. Let $\rho \in U_{\infty}$. Assume that $f \in W P \mathcal{S} A P_{\omega}(\mathbb{Z} \times$ $X, X, \rho)$ and there exists a constant $L_{f}>0$ such that

$$
\|f(k, u)-f(k, v)\| \leq L_{f}\|u-v\|, \quad \forall k \in \mathbb{Z}, u, v \in X .
$$

Then $\psi(\cdot)=f(\cdot, u(\cdot)) \in W P S A P_{\omega}(\mathbb{Z}, X, \rho)$ if $u \in$ $W P S A P_{\omega}(\mathbb{Z}, X, \rho)$.

\section{Nonautonomous Semilinear Difference Equations}

In this section, consider the following nonautonomous semilinear difference equations:

$$
u(n+1)=A(n) u(n)+f(n, u(n)), \quad n \in \mathbb{Z} .
$$

Its associated homogeneous linear difference equation is given by

$$
u(n+1)=A(n) u(n), \quad n \in \mathbb{Z} .
$$

To establish our results, we introduce the following conditions.

$\left(H_{1}\right)$ Let $f \in W P \mathcal{S} A P_{\omega}(\mathbb{Z} \times X, X, \rho), \rho \in U_{T}$.

$\left(H_{2}\right)$ There exists a constant $L_{f}>0$ such that

$$
\|f(k, u)-f(k, v)\| \leq L_{f}\|u-v\|, \quad \forall k \in \mathbb{Z}, u, v \in X .
$$

$\left(H_{2}^{\prime}\right)$ There exists a linear nondecreasing function $\Phi$ : $[0, \infty) \rightarrow[0, \infty)$ such that

$$
\|f(k, u)-f(k, v)\| \leq \Phi(\|u-v\|), \quad \forall k \in \mathbb{Z}, u, v \in X .
$$

$\left(H_{3}\right)$ Equation (34) admits an exponential dichotomy on $\mathbb{Z}$ with projection $P(n)$, positive constants $\eta, \nu, \alpha, \beta$.

$\left(H_{4}\right)$ For all $x \in X, \mathscr{A}(m+\omega, n+\omega) P(n+\omega) x=$ $\mathscr{A}(m, n) P(n) x$.

Theorem 14. Assume that $\left(H_{1}\right)-\left(H_{4}\right)$ hold and $\vartheta L_{f}<1$, where

$$
\vartheta:=\frac{\eta}{1-e^{-\alpha}}+\frac{\nu e^{-\beta}}{1-e^{-\beta}}
$$

then (33) has a unique solution $u(n) \in W P \mathcal{S} A P_{\omega}(\mathbb{Z}, X, \rho)$ which is given by

$$
\begin{aligned}
u(n)= & \sum_{j=-\infty}^{n-1} \mathscr{A}(n, j+1) P(j+1) f(j, u(j)) \\
& -\sum_{j=n}^{\infty} \mathscr{A}(n, j+1) Q(j+1) f(j, u(j)), \quad n \in \mathbb{Z} .
\end{aligned}
$$

Proof. Similarly as the proof of $[26,27]$, it can be shown that $u(\cdot)$ given by (38) is the solution of (33). Define the operator $\Gamma: W P S A P_{\omega}(\mathbb{Z}, X, \rho) \rightarrow W P S A P_{\omega}(\mathbb{Z}, X, \rho)$ as follows:

$$
\begin{aligned}
(\Gamma u)(n):= & \sum_{j=-\infty}^{n-1} \mathscr{A}(n, j+1) P(j+1) f(j, u(j)) \\
& -\sum_{j=n}^{\infty} \mathscr{A}(n, j+1) Q(j+1) f(j, u(j)), \quad n \in \mathbb{Z} .
\end{aligned}
$$

Since $u \in W P S A P_{\omega}(\mathbb{Z}, X, \rho)$ and $\left(H_{2}\right)$ holds, then $\Psi(\cdot)=$ $f(\cdot, u(\cdot)) \in W P S A P_{\omega}(\mathbb{Z}, X, \rho)$ by Corollary 13.

Next, we show that $\Gamma u \in W P S A P_{\omega}(\mathbb{Z}, X, \rho)$. In fact, let

$$
(\Gamma u)(n):=\left(\Gamma_{1} u\right)(n)-\left(\Gamma_{2} u\right)(n),
$$

where

$$
\begin{aligned}
& \left(\Gamma_{1} u\right)(n)=\sum_{j=-\infty}^{n-1} \mathscr{A}(n, j+1) P(j+1) \Psi(j), \\
& \left(\Gamma_{2} u\right)(n)=\sum_{j=n}^{\infty} \mathscr{A}(n, j+1) Q(j+1) \Psi(j) ;
\end{aligned}
$$


then,

$$
\begin{aligned}
& \frac{1}{\mu(n, \rho)} \sum_{k=-n}^{n} \rho(k)\left\|\left(\Gamma_{1} u\right)(k+\omega)-\left(\Gamma_{1} u\right)(k)\right\| \\
& =\frac{1}{\mu(n, \rho)} \sum_{k=-n}^{n} \rho(k) \| \sum_{j=-\infty}^{k+\omega-1} \mathscr{A}(k+\omega, j+1) P(j+1) \Psi(j) \\
& \quad-\sum_{j=-\infty}^{k-1} \mathscr{A}(k, j+1) P(j+1) \Psi(j) \| \\
& =\frac{1}{\mu(n, \rho)} \sum_{k=-n}^{n} \rho(k) \| \sum_{j=-\infty}^{k-1} \mathscr{A}(k, j+1) P(j+1) \\
& \leq \frac{1}{\mu(n, \rho)} \sum_{k=-n}^{n} \sum_{j=-\infty}^{k-1} \eta e^{-\alpha(k-j-1)} \rho(k)\|\Psi(j+\omega)-\Psi(j)\| \\
& =\frac{1}{\mu(n, \rho)} \sum_{k=-n}^{n} \sum_{j=0}^{\infty} \eta e^{-\alpha j} \rho(k) \| \Psi(k-1-j+\omega) \\
& =\sum_{j=0}^{\infty} \eta e^{-\alpha j}\left(\frac{1}{\mu(n, \rho)} \sum_{k=-n}^{n} \rho(k) \| \Psi(k-1-j+\omega)\right. \\
& -\Psi(k-1-j) \| \\
& -\Psi(k-1-j) \|) .
\end{aligned}
$$

Since $\Psi \in W P \mathcal{S} A P_{\omega}(\mathbb{Z}, X, \rho), \rho \in U_{T}$, then $\Psi(\cdot-1-j) \in$ $W P \mathcal{S} A P_{\omega}(\mathbb{Z}, X, \rho)$ for each $j \in \mathbb{Z}$ by Lemma 10 ; hence

$$
\begin{aligned}
\lim _{n \rightarrow \infty} & \frac{1}{\mu(n, \rho)} \sum_{k=-n}^{n} \rho(k)\|\Psi(k-1-j+\omega)-\Psi(k-1-j)\| \\
& =0 .
\end{aligned}
$$

By Lebesgue dominated convergence theorem, one has

$$
\begin{array}{r}
\lim _{n \rightarrow \infty} \frac{1}{\mu(n, \rho)} \sum_{k=-n}^{n} \rho(k)\left\|\left(\Gamma_{1} u\right)(k+\omega)-\left(\Gamma_{1} u\right)(k)\right\| \\
\leq \sum_{j=0}^{\infty} \eta e^{-\alpha j}\left(\lim _{n \rightarrow \infty} \frac{1}{\mu(n, \rho)} \sum_{k=-n}^{n} \rho(k) \| \Psi(k-1-j+\omega)\right. \\
-\Psi(k-1-j) \|)
\end{array}
$$$$
=0 \text {; }
$$

hence $\Gamma_{1} u \in W P \mathcal{S} A P_{\omega}(\mathbb{Z}, X, \rho)$. Similarly, one can prove $\Gamma_{2} u \in W P S A P_{\omega}(\mathbb{Z}, X, \rho)$. So $\Gamma$ is well defined.

For $u, v \in W P \mathcal{S} A P_{\omega}(\mathbb{Z}, X, \rho)$, by $\left(H_{2}\right)$ and exponential dichotomy, one has

$$
\begin{aligned}
&\|(\Gamma u)(n)-(\Gamma v)(n)\| \\
&=\| \sum_{j=-\infty}^{n-1} \mathscr{A}(n, j+1) P(j+1)[f(j, u(j))-f(j, v(j))] \\
& \quad-\sum_{j=n}^{\infty} \mathscr{A}(n, j+1) Q(j+1)[f(j, u(j))-f(j, v(j))] \| \\
& \leq \sum_{j=-\infty}^{n-1} \eta e^{-\alpha(n-j-1)} L_{f}\|u(j)-v(j)\| \\
& \quad+\sum_{j=n}^{\infty} \nu \mathrm{e}^{-\beta(j+1-n)} L_{f}\|u(j)-v(j)\| \\
& \leq \eta L_{f}\|u-v\|_{d} \sum_{j=0}^{\infty} e^{-\alpha j}+v L_{f}\|u-v\|_{d} \sum_{j=1}^{\infty} e^{-\beta j} \\
& \leq \frac{\eta L_{f}}{1-e^{-\alpha}}\|u-v\|_{d}+\frac{v L_{f} e^{-\beta}}{1-e^{-\beta}}\|u-v\|_{d}=\vartheta L_{f}\|u-v\|_{d} ;
\end{aligned}
$$

hence $\Gamma$ is a contraction. By the Banach contraction mapping principle, $\Gamma$ has a unique fixed point $u \in W P \mathcal{S} A P_{\omega}(\mathbb{Z}, X, \rho)$, which is the unique $W P \mathcal{S} A P_{\omega}$ solution of (33). The proof is completed.

Theorem 15. Assume that $\left(H_{1}\right),\left(H_{2}^{\prime}\right),\left(H_{3}\right)$, and $\left(H_{4}\right)$ hold; then (33) has a unique solution $u(n) \in W P \mathcal{S} A P_{\omega}(\mathbb{Z}, X, \rho)$ if $(\vartheta \Phi)^{n}(t) \rightarrow 0$ as $n \rightarrow \infty$ for each $t>0$.

Proof. Define the operator $\Gamma$ as in (39), so $\Gamma$ is well defined. For $u, v \in W P \mathcal{S} A P_{\omega}(\mathbb{Z}, X, \rho)$, one has

$$
\begin{aligned}
&\|(\Gamma u)(n)-(\Gamma v)(n)\| \\
& \leq \sum_{j=-\infty}^{n-1} \eta e^{-\alpha(n-j-1)}\|f(j, u(j))-f(j, v(j))\| \\
& \quad+\sum_{j=n}^{\infty} v e^{-\beta(j+1-n)}\|f(j, u(j))-f(j, v(j))\| \\
& \leq \sum_{j=-\infty}^{n-1} \eta e^{-\alpha(n-j-1)} \Phi(\|u(j)-v(j)\|) \\
& \quad+\sum_{j=n}^{\infty} v e^{-\beta(j+1-n)} \Phi(\|u(j)-v(j)\|) \\
& \leq \Phi(\|u-v\|) \sum_{j=0}^{\infty} \eta e^{-\alpha j}+\Phi(\|u-v\|) \sum_{j=1}^{\infty} v e^{-\beta j} \\
& \leq \vartheta \Phi(\|u-v\|) .
\end{aligned}
$$


Since $(\vartheta \Phi)^{n}(t) \rightarrow 0$ as $n \rightarrow \infty$ for each $t>0$, by Matkowski fixed point theorem (Theorem 1), $\Gamma$ has a unique fixed point $u \in W P S A P_{\omega}(\mathbb{Z}, X, \rho)$, which is the unique $W P \mathcal{S} A P_{\omega}$ solution of (33).

Example 16. Consider the system

$$
u(n+1)=A u(n)+h(n) g(u), \quad n \in \mathbb{Z},
$$

where $A$ is a nonsingular $k \times k$ matrix such that $\sigma_{p}(A) \cap S^{1}=$ $\emptyset, h \in W P \mathcal{S} A P_{\omega}\left(\mathbb{Z}, \mathbb{R}^{k}, \rho\right)$, and $\rho \in U_{T}$ and there exists a constant $L_{g}>0$ such that

$$
\|g(u)-g(v)\| \leq L_{g}\|u-v\|, \quad u, v \in \mathbb{R}^{k} .
$$

Since $\sigma_{p}(A) \cap S^{1}=\emptyset$, the system

$$
u(n+1)=A u(n), \quad n \in \mathbb{Z}
$$

admits an exponential dichotomy with positive constants $\eta, \nu, \alpha, \beta$ [25] and $\left(\mathrm{H}_{2}\right)$ holds with $L_{f}=L_{g}\|h\|_{d}$. By Theorem 14, if we suppose that $\vartheta L_{g}\|h\|_{d}<1$, then (47) has a unique discrete weighted pseudo- $\mathcal{S}$-asymptotically $\omega$ periodic solution.

\section{Scalar Second Order Difference Equations}

Let $X=\mathbb{R}$; we study the existence and uniqueness of discrete weighted pseudo- $\mathcal{S}$-asymptotically $\omega$-periodic solutions to a scalar second order difference equation given by

$$
\begin{aligned}
& u(n+2)+b(n) u(n+1)+a(n) u(n) \\
& =f(n, u(n)), \quad n \in \mathbb{Z},
\end{aligned}
$$

where $f \in W P \mathcal{S} A P_{\omega}(\mathbb{Z} \times \mathbb{R}, \mathbb{R}, \rho)$ and $\rho \in U_{T}$ and satisfies the following.

$\left(A_{1}\right)$ The function $(n, x) \rightarrow f(n, x)$ is Lipschitz in $x \in \mathbb{R}^{2}$ uniformly in $n \in \mathbb{Z}$; that is, there exists a constant $L_{f}>0$ such that

$$
\|f(n, x)-f(n, y)\| \leq L_{f}\|x-y\| \quad x, y \in \mathbb{R}^{2}, n \in \mathbb{Z} .
$$

$\left(A_{2}\right) a, b: \mathbb{Z} \rightarrow \mathbb{R}$ are periodic functions, in the sense that there exists $\omega \in \mathbb{Z}^{+}$such that

$$
a(n+\omega)=a(n), \quad b(n+\omega)=b(n), \quad n \in \mathbb{Z} .
$$

$\left(A_{3}\right)$ There exist $a_{0}, b_{0}>0$ such that $\inf _{n \in \mathbb{Z}} a(n)=a_{0}$ and $\inf _{n \in \mathbb{Z}} b(n)=b_{0}$.

$\left(A_{4}\right) \operatorname{Let} b(n) \neq 2 \sqrt{a(n)}$ for all $n \in \mathbb{Z}$.

Now, let

$$
A(n)=\left(\begin{array}{cc}
0 & 1 \\
-a(n) & -b(n)
\end{array}\right), \quad n \in \mathbb{Z}
$$

then (50) can be rewritten as the abstract form (33). It is not difficult to see that

$$
\operatorname{det}\left(A(n)-\lambda I_{\mathbb{R}^{2}}\right)=\lambda^{2}+b(n) \lambda+a(n), \quad n \in \mathbb{Z} .
$$

Let $D(n)=b^{2}(n)-4 a(n)$ for all $n \in \mathbb{Z}$; then $\left(A_{4}\right)$ implies that either $D(n)>0$ or $D(n)<0$ for all $n \in \mathbb{Z}$. By $\left(A_{3}\right)$ and $\left(A_{4}\right)$, one has what follows.

(1) If $D(n)>0$ for all $n \in \mathbb{Z}$, then the eigenvalues of $A(n)$ are given by

$$
\begin{aligned}
& \lambda_{1}(n)=\frac{-b(n)+\sqrt{b^{2}(n)-4 a(n)}}{2}, \\
& \lambda_{2}(n)=\frac{-b(n)-\sqrt{b^{2}(n)-4 a(n)}}{2} .
\end{aligned}
$$

Moreover, it can be easily shown that $\lambda_{1}(n)<0, \lambda_{2}(n)<0$ for all $n \in \mathbb{Z}$.

(2) If $D(n)<0$ for all $n \in \mathbb{Z}$, then the eigenvalues of $A(n)$ are given by

$$
\begin{aligned}
& \lambda_{1}(n)=\frac{-b(n)+i \sqrt{4 a(n)-b^{2}(n)}}{2}, \\
& \lambda_{2}(n)=\frac{-b(n)-i \sqrt{4 a(n)-b^{2}(n)}}{2} .
\end{aligned}
$$

Moreover, it can be easily shown that $\mathfrak{R e} \lambda_{1}(n)<$ $0, \mathfrak{R e} \lambda_{2}(n)<0$ for all $n \in \mathbb{Z}$.

In view of the above, it follows that the homogeneous linear difference equation

$$
u(n+1)=A(n) u(n), \quad n \in \mathbb{Z},
$$

has an exponential dichotomy on $\mathbb{Z}[28]$. Since $A(n+\omega)=$ $A(n),\left(H_{4}\right)$ holds. By Theorem 14, one has the following.

Theorem 17. Under assumptions $\left(A_{1}\right)-\left(A_{4}\right)$, (50) has a unique solution $u(n) \in W P S A P_{\omega}(\mathbb{Z}, \mathbb{R}, \rho)$ whenever $L_{f}$ is small enough.

\section{Conflict of Interests}

The author declares that there is no conflict of interests regarding the publication of this paper.

\section{Acknowledgment}

This research was supported by Zhejiang Provincial Natural Science Foundation of China under Grant no. LQ13A010015.

\section{References}

[1] H. R. Henríquez, M. Pierri, and P. Táboas, "On S-asymptotically $\omega$-periodic functions on Banach spaces and applications," Journal of Mathematical Analysis and Applications, vol. 343, no. 2, pp. 1119-1130, 2008. 
[2] H. R. Henríquez, M. Pierri, and P. Táboas, "Existence of $\mathcal{S}$-asymptotically $\omega$-periodic solutions for abstract neutral functional-differential equations," Bulletin of the Australian Mathematical Society, vol. 78, no. 3, pp. 365-382, 2008.

[3] F. Y. Wei and K. Wang, "An asymptotically periodic logistic equation," Journal of Biomathematics, vol. 20, no. 4, pp. 399-405, 2005.

[4] J. Blot, P. Cieutat, and G. M. N’Guérékata, "S-asymptotically $\omega$-periodic functions and applications to evolution equations," African Diaspora Journal of Mathematics, vol. 12, no. 2, pp. 113121, 2011.

[5] C. Cuevas and J. C. de Souza, " $S$-asymptotically $\omega$-periodic solutions of semilinear fractional integro-differential equations," Applied Mathematics Letters, vol. 22, no. 6, pp. 865-870, 2009.

[6] C. Cuevas and C. Lizama, "S-asymptotically $\omega$-periodic solutions for semilinear Volterra equations," Mathematical Methods in the Applied Sciences, vol. 33, no. 13, pp. 1628-1636, 2010.

[7] B. de Andrade and C. Cuevas, "S-asymptotically $\omega$-periodic and asymptotically $\omega$-periodic solutions to semi-linear Cauchy problems with non-dense domain," Nonlinear Analysis: Theory, Methods and Applications, vol. 72, no. 6, pp. 3190-3208, 2010.

[8] W. Dimbour, G. Mophou, and G. M. N’Guérékata, "Sasymptotically periodic solutions for partial differential equations with finite delay," Electronic Journal of Differential Equations, vol. 2011, no. 117, pp. 1-12, 2011.

[9] J. P. C. dos Santos and S. M. Guzzo, "Solutions in several types of periodicity for partial neutral integro-differential equation," Electronic Journal of Differential Equations, vol. 2013, no. 31, pp. $1-18,2013$.

[10] M. Pierri, "On S-asymptotically $\omega$-periodic functions and applications," Nonlinear Analysis: Theory, Methods and Applications, vol. 75, no. 2, pp. 651-661, 2012.

[11] M. Pierri and V. Rolnik, "On pseudo $S$-asymptotically periodic functions," Bulletin of the Australian Mathematical Society, vol. 87, no. 2, pp. 238-254, 2013.

[12] Z. N. Xia, "Weighted pseudo asymptotically periodic solutions for differential and integro-differential equations in Banach spaces," Submitted.

[13] R. P. Agarwal, C. Cuevas, and M. V. S. Frasson, "Semilinear functional difference equations with infinite delay," Mathematical and Computer Modelling, vol. 55, no. 3-4, pp. 1083-1105, 2012.

[14] S. K. Choi and N. Koo, "Almost periodic solutions of nonlinear discrete Volterra equations with unbounded delay," Advances in Difference Equations, vol. 2008, Article ID 692713, 15 pages, 2008.

[15] Y. Song and H. Tian, "Periodic and almost periodic solutions of nonlinear discrete Volterra equations with unbounded delay," Journal of Computational and Applied Mathematics, vol. 205, no. 2, pp. 859-870, 2007.

[16] L. Del Campo, M. Pinto, and C. Vidal, "Almost and asymptotically almost periodic solutions of abstract retarded functional difference equations in phase space," Journal of Difference Equations and Applications, vol. 17, no. 6, pp. 915-934, 2011.

[17] Y. Song, "Asymptotically almost periodic solutions of nonlinear Volterra difference equations with unbounded delay," Journal of Difference Equations and Applications, vol. 14, no. 9, pp. 971-986, 2008.

[18] R. P. Agarwal, C. Cuevas, and F. Dantas, "Almost automorphy profile of solutions for difference equations of Volterra type," Journal of Applied Mathematics and Computing, vol. 42, no. 12, pp. 1-18, 2013.
[19] A. Castro, C. Cuevas, F. Dantas, and H. Soto, "About the behavior of solutions for Volterra difference equations with infinite delay," Journal of Computational and Applied Mathematics, vol. 255, pp. 44-59, 2014.

[20] C. Cuevas, H. R. Henríquez, and C. Lizama, "On the existence of almost automorphic solutions of Volterra difference equations," Journal of Difference Equations and Applications, vol. 18, no. 11, pp. 1931-1946, 2012.

[21] C. Cuevas, F. Dantas, and M. Choquehuanca, "I ${ }^{P}$-boundedness properties for Volterra difference equations," Applied Mathematics and Computation, vol. 219, no. 12, pp. 6986-6999, 2013.

[22] F. L. Chen and Y. Zhou, "Existence and Ulam stability of solutions for discrete fractional boundary value problem," Discrete Dynamics in Nature and Society, vol. 2013, Article ID 459161, 7 pages, 2013.

[23] M. Liao, "Global asymptotic stability of a family of nonlinear difference equations," Discrete Dynamics in Nature and Society, vol. 2013, Article ID 750852, 4 pages, 2013.

[24] J. Matkowski, "Integrable solutions of functional equations," Dissertationes Mathematicae, vol. 127, pp. 1-68, 1975.

[25] R. P. Agarwal, Difference Equations and Inequalities: Theory Methods and Applications, Marcel Dekker, New York, NY, USA, 1992.

[26] T. Diagana, "Existence of globally attracting almost automorphic solutions to some nonautonomous higher-order difference equations," Applied Mathematics and Computation, vol. 219, no. 12, pp. 6510-6519, 2013.

[27] C. Lizama and J. G. Mesquita, "Almost automorphic solutions of non-autonomous difference equations," Journal of Mathematical Analysis and Applications, vol. 407, no. 2, pp. 339-349, 2013.

[28] D. Henry, Geometric Theory of Semilinear Parabolic Equations, vol. 840 of Lecture Notes in Mathematics, Springer, New York, NY, USA, 1981. 


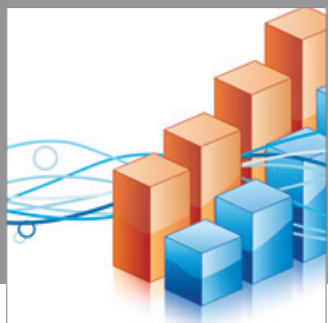

Advances in

Operations Research

mansans

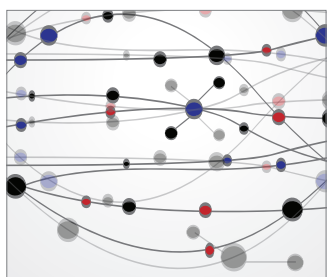

The Scientific World Journal
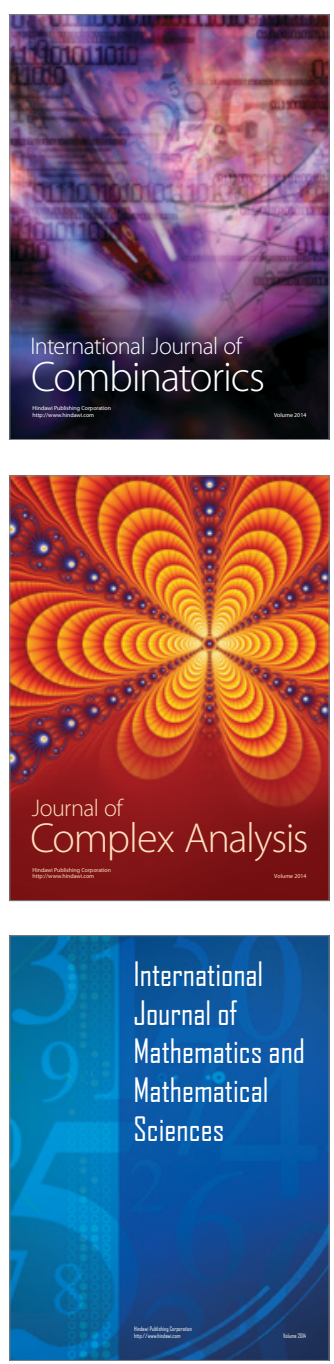
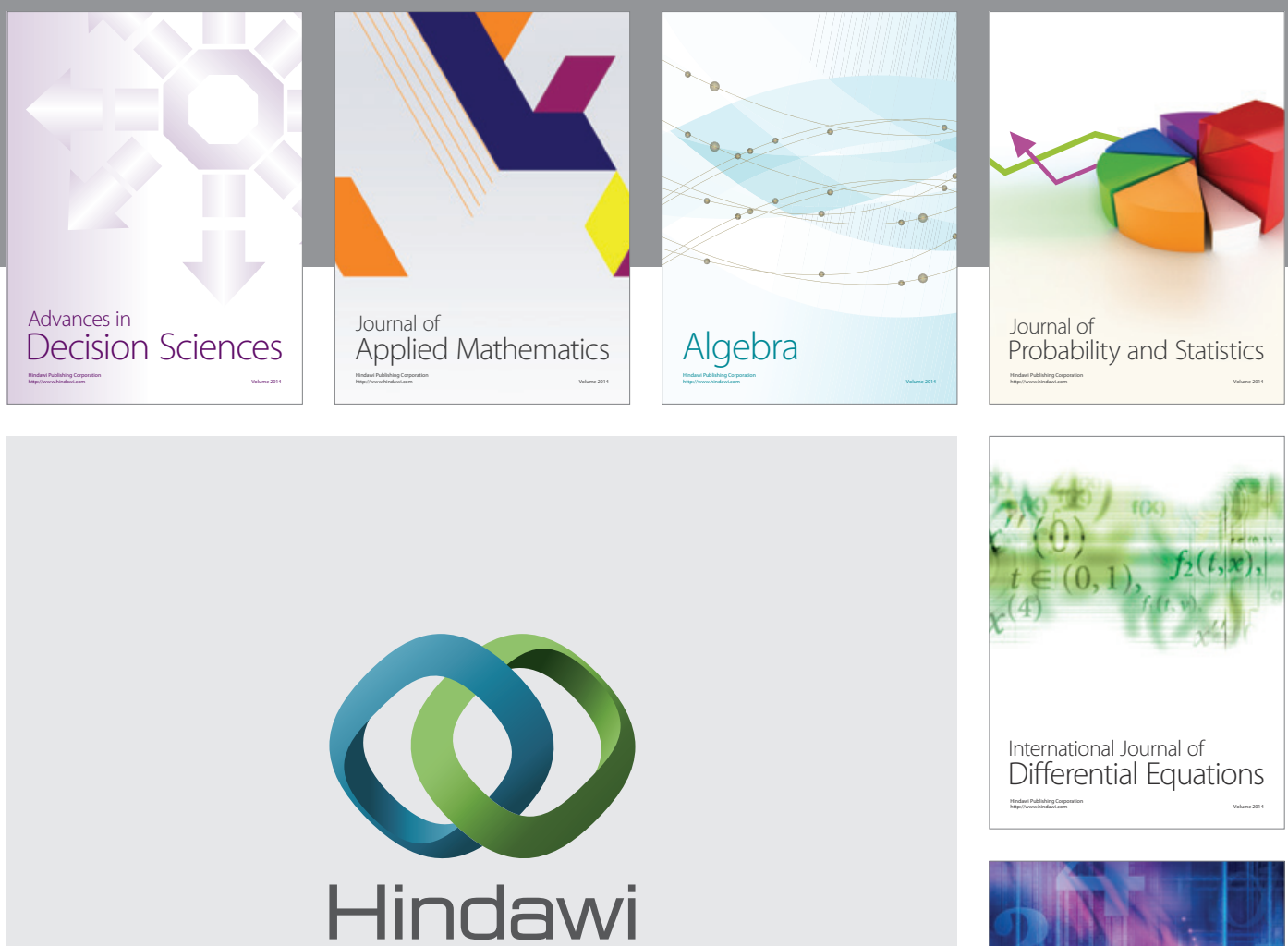

Submit your manuscripts at http://www.hindawi.com
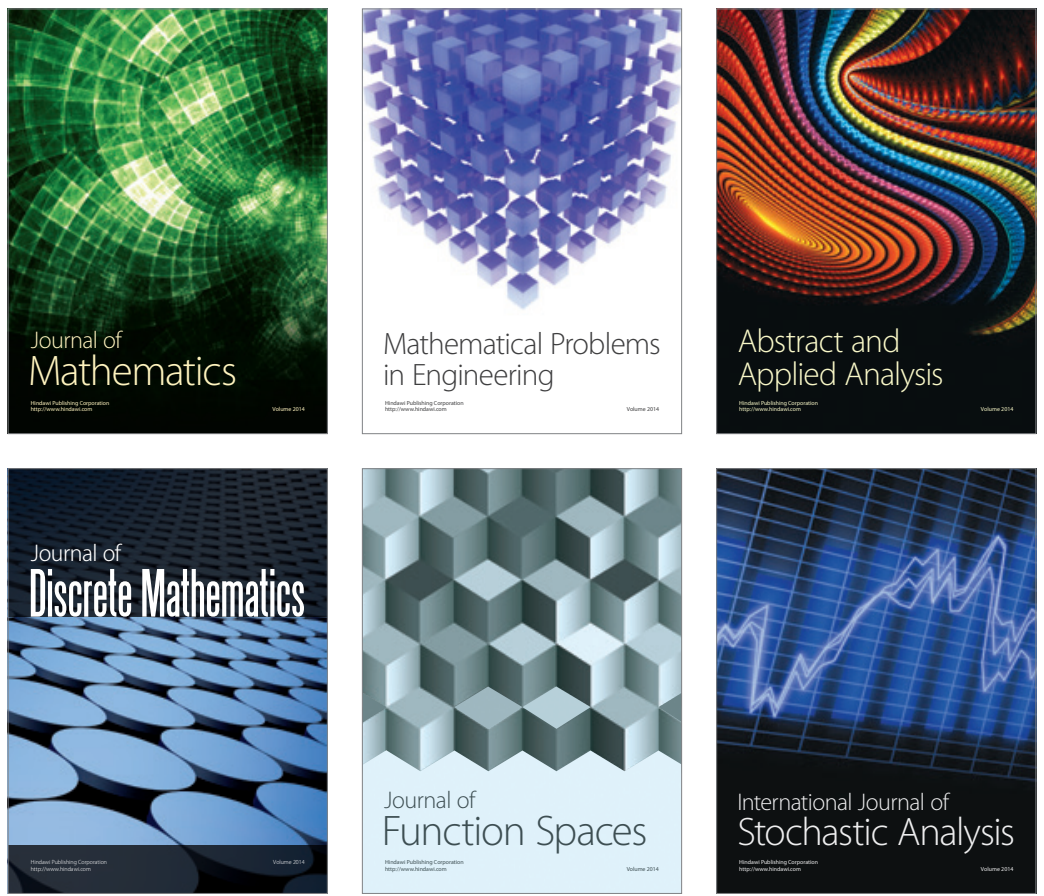

Journal of

Function Spaces

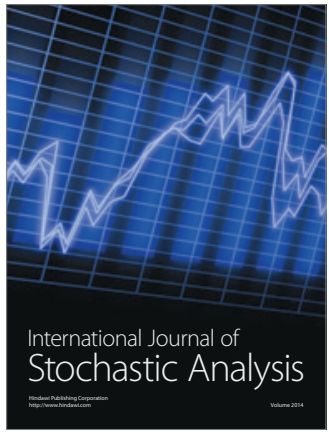

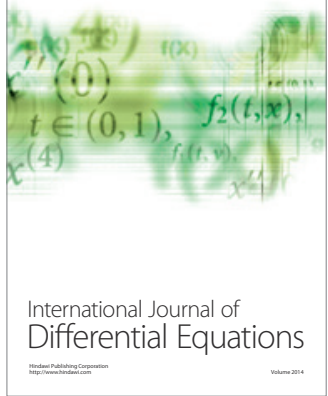
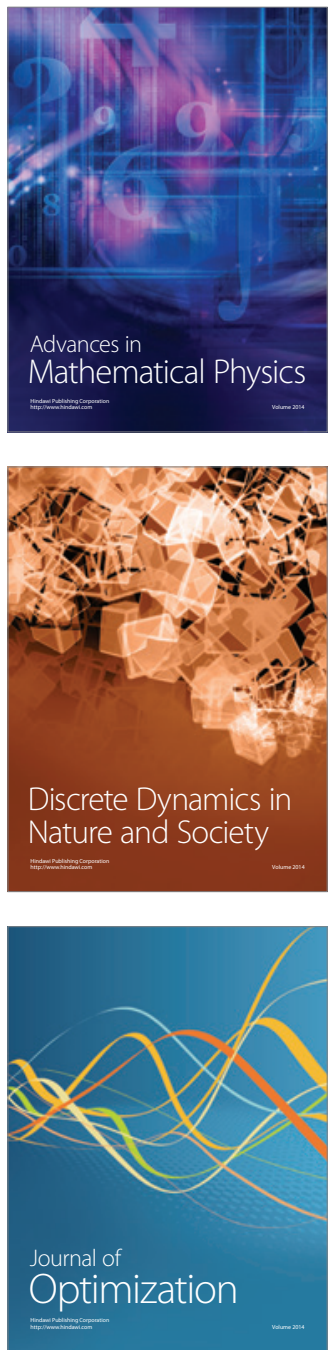